
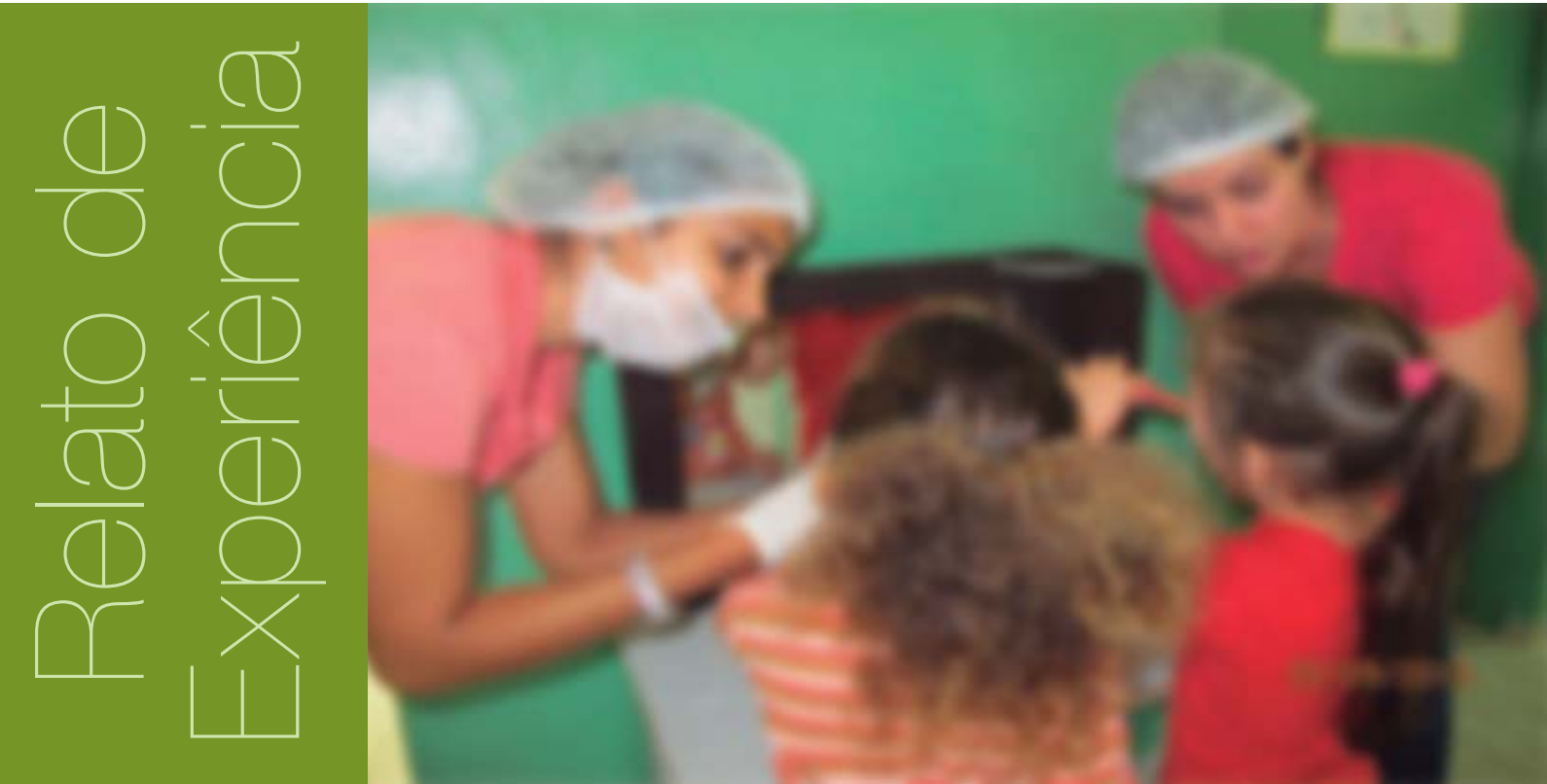

\title{
Tratamento Restaurador Atraumático: da experiência assistencial à capacitação em saúde pública
}

Antônio Fernando Monnerat ${ }^{1}$ - antoniofmonnerat@gmail.com

Maria Isabel de Castro de Souza ${ }^{1}$ - profamariaisabel@yahoo.com.br

Aline Borges Luiz Monnerat² - alinelmonnerat@gmail.com

\section{RESUMO}

Este texto pretende apresentar a experiência do Projeto de Extensão "Tratamento Restaurador Atraumático (TRA)", iniciado em março de 2008, com o objetivo de levar graduandos da Faculdade de Odontologia da UERJ para Resende e Itatiaia (RJ), atuando por meio do TRA associado à educação para a saúde, em comunidades com difícil acesso à odontologia. Com resultados expressivos, em 2011, o projeto passou também a capacitar profissionais de saúde pública em grandes centros e no interior de todo o país. A partir da experiência adquirida, 0 Projeto de Extensão desenvolveu um Curso de Capacitação em TRA por meio do Teleodontologia para profissionais de saúde e livro texto.

\section{PALAVRAS-CHAVE}

Odontologia. Saúde Pública. Tratamento Restaurador Atraumático. Promoção de Saúde. Cárie.

\section{ABSTRACT}

This paper aims to present the experience of the Extension Project "Atraumatic Restorative Treatment (ART)", initiated in March 2008, with the goal of bringing students from the Faculty of Dentistry, UERJ to Resende and Itatiaia (RJ), acting through ART together with Health promotion and education in communities with poor access to dentistry. With significant results, the project,

\footnotetext{
1 Professor Associado do Departamento de Dentística da Faculdade de Odontologia UERJ

2 Doutoranda em Periodontia da Faculdade de Odontologia da UERJ
} 
in 2011, also began technical training on public health professionals in major centers and within the whole country. The Extension Project developed from the experience gained, Training Course on TRA through Teledentistry for health professionals and textbook.

\section{KEYWORDS}

Dentistry. Public Health. Atraumatic Restorative Treatment (ART). Health Promotion. Caries.

\section{Relato de experiência}

Atualmente nos grandes centros, os alunos de graduação têm dificuldades de vivenciar realidades diferentes daquelas que encontram ao atender pacientes na universidade. Tal fato ocorre devido a fatores como violência e dificuldade de acesso a algumas comunidades. Com o intuito de fazê-los vivenciar uma experiência com comunidades rurais e urbanas com vulnerabilidade socioeconômica e promover a interiorização das ações extensionistas da universidade, surgiu o Projeto de Extensão Tratamento Restaurador Atraumático, da Faculdade de Odontologia da Universidade do Estado do Rio de Janeiro. 0 projeto foi realizado nos municípios de Resende e Itatiaia-RJ.

Tais municípios estão localizados na região sul do Estado do Rio de Janeiro e compreendem juntos uma população de aproximadamente 250.000 habitantes. 0 recente crescimento populacional e econômico da região é considerado único na história.

Há aproximadamente 15 anos, fábricas automotivas lá se instalaram e com elas, outras vieram para suportá-las, além de empresas de serviços e construção civil, resultando na criação de 7000 empregos diretos. Este crescimento resultou em uma incapacidade de atendimento básico e as comunidades ali residentes, continuam ainda totalmente desassistidas.

Neste contexto, alguns bairros de Resende e de municípios vizinhos como Itatiaia não têm em sua proximidade postos de saúde que seriam capazes de receber essa população, que quando pode, viaja a pé ou pagando transporte público, por mais de uma hora para ter atendimento em ambientes de saúde em comunidades vizinhas.

Neste contexto, o Projeto de Extensão em Tratamento Restaurador Atraumático iniciou suas atividades em 2008 visando atender comunidades destes municípios (Resende e Itatiaia), priorizando crianças e gestantes, mas atendendo na medida do possível pais e familiares dos mesmos.

Os locais de execução do projeto compreenderam: uma creche com crianças de 0 a 4 anos da entidade filantrópica Casa da Amizade (Resende); comunidade rural "Terra Livre" (Resende); comunidade Lavapés (Resende); comunidade de Penedo em ambiente odontológico na Associação Centro de Estudos Odontológicos Penedo (Itatiaia); Escola Municipal Sebastião Antônio Bernardes (Itatiaia).

0 projeto TRA Resende atuou sempre às sextas-feiras. Os alunos e voluntários saíam do Rio de Janeiro às 06h30min e retornavam às $19 \mathrm{~h}$.

No atendimento, os pacientes respondiam a um questionário de saúde (anamnese), seguido de um exame clínico onde identificava-se e registrava-se: Índice de Placa Visível Simplificado (IPV); Índice de Sangramento Gengival Simplificado (ISG) e Índice CPOD. Estes índices tem objetivo de diagnosticar o perfil epidemiológico da população participante, bem como avaliar a eficácia do projeto. Não houve critério de exclusão. Foram priorizados os atendimentos a crianças, gestantes e pais, nesta ordem. No caso do atendimento em ambiente não odontológico, carteiras escolares foram utilizadas como bancadas de atendimento.

Com auxílio de lanternas e utilizando luz natural, os exames clínicos foram realizados. Com relação aos instrumentos, instrumentais para exame clínico e o Kit de instrumental específico para TRA da empresa SS White foram utilizados. 
Na consulta seguinte ao primeiro encontro, o paciente e o responsável - no caso de menores de idade - recebiam palestra educativa e instrução de higiene oral individualizada. 0 paciente, quando necessário, recebia o tratamento restaurador atraumático por quadrantes. Após a conclusão do tratamento o paciente recebia alta sendo convocado para revisão dos mesmos índices após seis meses.

0 Tratamento Restaurador Atraumático atua nos conceitos de técnica minimamente invasiva, permitindo manutenção de estrutura dental sadia através da remoção seletiva de cárie com instrumentos manuais e restauração com Cimento de lonômero de Vidro (CIV) de alta viscosidade. Como uma das suas vantagens, há redução do número de exposições pulpares, reduzindo desta forma endodontias e exodontias. Há também menor estresse e ansiedade do paciente, visto que raramente causa dor, não necessitando de anestesia, o que é relevante na odontopediatria. 0 método tem sido descrito como econômico e eficaz na prevenção e controle da doença da cárie em populações em situação de vulnerabilidade socioeconômica.

Como já descrito anteriormente, o projeto atuou ou atua em cinco ambientes diferentes que são apresentados a seguir:

1. Creche Casa da Amizade, Resende (Figuras 1a e 1b) - uma manhã por mês. A atuação principal é na prevenção de cárie e conscientização de professores e pais da importância da Saúde Bucal. Quando necessário, a equipe também restaura pela técnica TRA. Foram atendidos 238 pacientes crianças entre zero e quatro anos. Os profissionais envolvidos com a educação escolar e o gestor da Creche se mostraram muito interessados nas informações e nas práticas desenvolvidas nesse ambiente 0 que facilitou e facilita sobremaneira 0 trabalho desenvolvido $e$ a continuidade das ações educativas em saúde. Neste local, o projeto atua desde 2008 até os dias atuais;

2. Comunidade Terra Livre, Resende (Figuras 2a e 2b) - um dia por mês. Esta comunidade é classificada como rural e o projeto teve sua atuação sem tecnologia odontológica. Foram atendidos 98 pacientes entre crianças e adultos. A dificuldade apresentada nessa localidade diz respeito, em sua maioria, à ausência dos responsáveis nas ações em saúde. Somente a professora da escola rural e um número reduzido de pais participaram do processo. A ausência de um líder comunitário dificultou o comprometimento e a aceitação ao projeto nesse local. 0 projeto atua desde 2008 até os dias atuais.

3. Comunidade Lavapés - Casa Espírita Joana de Angelis, Resende - uma tarde por mês. Atuação sem tecnologia odontológica. Foram atendidos 313 pacientes, entre crianças e adultos (zero a 18 anos). 0 projeto funcionou de 2009 a 2011.

4. Clínica do CEO Penedo, Itatiaia (duas vezes ao mês). É o único local com clínica odontológica do projeto. Neste local foram atendidos pacientes residentes no bairro e casos complexos das demais localidades. Desde 2008 até os dias atuais foram atendidos 800 pacientes de todas as faixas etárias.

5. Escola Municipal Sebastião Antonio Bernardes em Penedo, Itatiaia (Figuras 3a e 3b). A Escola possui 180 alunos. Foram realizados levantamento epidemiológico das doenças bucais, instrução de higiene oral, palestras para pais e professores e ações educativas com as crianças. A direção da escola mostrou-se muito interessada e reconheceu a relevância do projeto de extensão, o que contribui para excelente adesão e atuação do projeto no local. As atividades foram iniciadas em 2013 e compreendem atendimento a pacientes de cinco a 14 anos.

Figuras 1A e 1B: Creche da Casa da Amizade.

Fonte: Imagens dos autores.

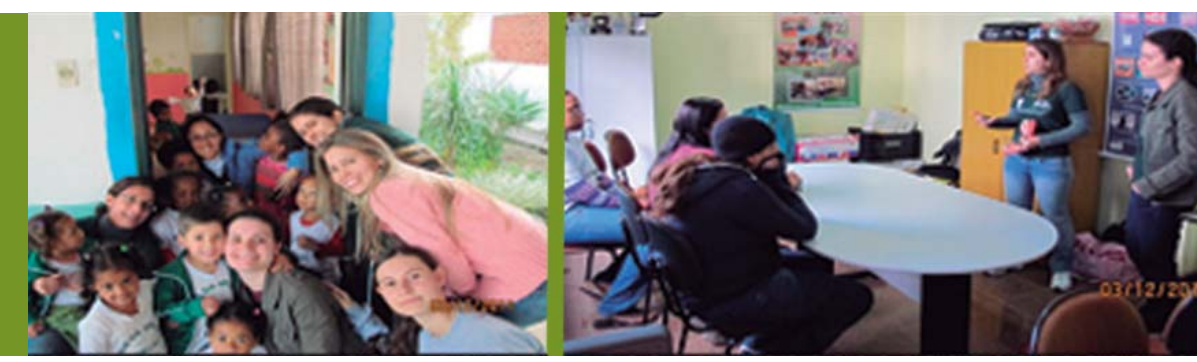




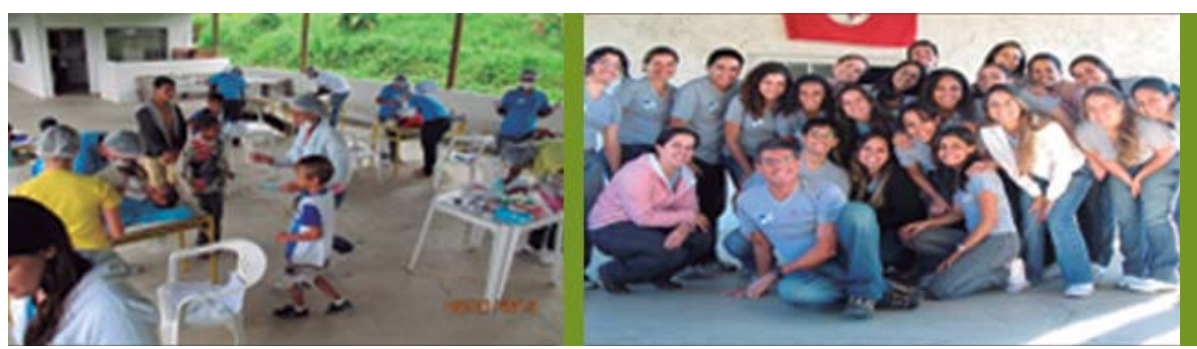

Figuras 2A e 2B:

Comunidade Terra Livre

Fonte: Imagens dos autores.
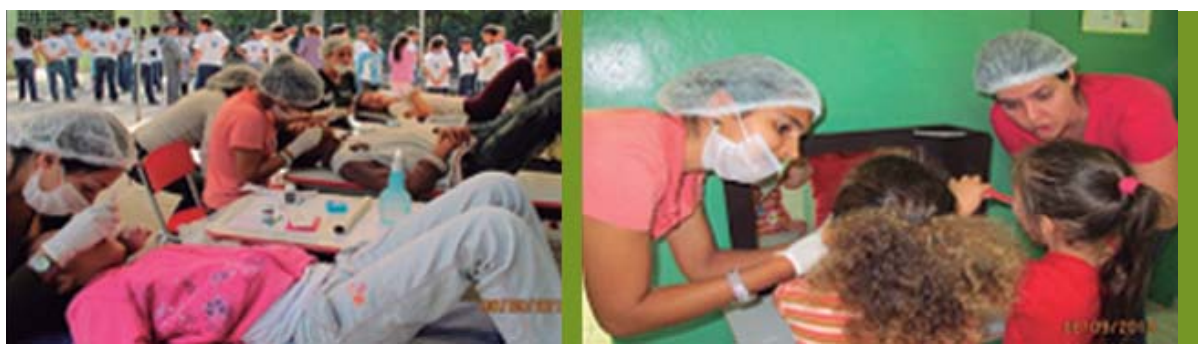

Figuras 3A e 3B: Escola

Sebastião Bernardo.

Fonte: Imagens dos autores.

Além do atendimento nestes locais, a partir de 2011, o projeto apostou na Educação Continuada, seguindo a literatura científica, na qual uma das barreiras à implementação do TRA seria a falta de capacitação, treinamento e conhecimento técnico científico.

Inicialmente, o Projeto de Extensão em TRA objetivou a capacitação de profissionais de odontologia das redes públicas. Utilizou-se a plataforma do Telessaúde Redes Brasil - Núcleo Rio de Janeiro (Teleodontologia) para alocar o Curso de Capacitação em TRA. Sob a forma de capacitação teórica foi divulgado em serviços de saúde pública em todo o Brasil. Há relatos de alunos das regiões Sudeste, Norte e Nordeste que concluíram o curso de 10 módulos (15 horas/ aula).

A Teleodontologia permite a disseminação da atualização científica e seminários (ao vivo) para todo o país através de um programa do governo federal, o Telessaúde. 0 núcleo Rio de Janeiro encontra-se no Hospital Universitário Pedro Ernesto (HUPE) na Universidade do Estado do Rio de Janeiro.

A metodologia do Ensino à Distância (EAD) permite que o profissional realize a discussão científica ou a atualização de qualquer lugar e em qualquer horário. A partir de relatos científicos considera-se que a capacitação é uma ferramenta essencial para divulgar o TRA na classe odontológica desmistificando a técnica e incentivando sua utilização, principalmente em saúde pública.

Paralelamente a isso, foi oferecida à Secretaria Municipal de Saúde do Município do Rio de Janeiro (SMSDC/RJ), a capacitação de profissionais que atuam na Atenção Primária e Equipes de Saúde da Família. Neste caso, participaram do curso 500 dentistas, o qual compreendeu aulas teóricas e práticas em escolas e comunidades. Outros 900 profissionais em diferentes municípios do Brasil receberam a capacitação, a saber:

Curso de Extensão para 24 alunos da Graduação da F0-UERJ em janeiro de 2013; Curso de Capacitação Teórico e Prático na técnica TRA para 48 dentistas, 22 Técnicos em saúde Bucal (TSB) e 20 Auxiliares em saúde Bucal (ASB) da Prefeitura Municipal de São José do Rio Preto, SP em março e agosto de 2013; Curso de Capacitação Teórico e Prático na técnica TRA para 37 dentistas, 18 TSBs e 25 ASBs da Prefeitura Municipal de Limeira, SP em março setembro de 2013; Capacitações Teóricas (8 hs) em TRA para 720 profissionais em: São João de Meriti, RJ; Limeira, SP; Barretos, SP; Belém; PA; São José dos Pinhais, PR; Campos, RJ e Niterói, RJ.

Para os membros do projeto, acredita-se ser fundamental o aprimoramento técnico e discussão científica, além de padronização para trabalhar com a técnica. Com as capacitações, reduzemse as falhas técnicas, se promove a divulgação do TRA preconizado pela OMS e amplia-se 0 atendimento dentro deste protocolo. 
Uma vez que os profissionais capacitados atuem com a coletividade, na saúde pública e em muitos casos nas situações de comunidades de difícil acesso ou ausência de postos de saúde (Equipes de Saúde da Família), é possível ampliar a assistência e eficácia na redução da Doença Cárie e melhora dos níveis de saúde oral da população contemplada.

Diante da experiência dos membros da equipe do projeto, objetivou-se criar uma literatura de apoio ao profissional que atua em saúde pública. Em 2012 o Projeto de Extensão em TRA iniciou a elaboração de um livro intitulado: "Restaurações em Saúde Pública" com 12 capítulos. 0 aceite para a publicação pela Editora Elsevier se deu em 2013 e o lançamento previsto para segundo semestre de 2014. 0 livro cria uma atmosfera de atualização científica dentro do universo do TRA. Desde a revisão da literatura, descrição da técnica, materiais dentários de escolha, descrição detalhada da técnica, sua utilização na Odontopediatria, Odontogeriatria, em Pacientes Especiais, em Gestantes, em pacientes com Câncer entre outros grupos. É possível extrair do livro a organização de insumos, montagem do protocolo personalizado a cada ambiente social, administração da equipe de trabalho para atuar com o TRA em uma comunidade específica ou ambiente determinado.

Novas perspectivas são construídas para o Projeto de Extensão em TRA. Em 2014, inicia-se atividades de promoção de saúde baseada na prevenção das principais doenças bucais no grupo de gestantes de alto risco do Ambulatório de Pré-Natal do Hospital Universitário Pedro Ernesto da Universidade do Estado do Rio de Janeiro (HUPE- UERJ).

Acreditando ser esse grupo (gestantes) um excelente grupo para abordagem preventiva, o Projeto de Extensão em TRA atua no pré-natal em parceria com a equipe médica e enfermagem, reduzindo assim o risco de acometimento da doença cárie no infante e melhorando os níveis de saúde bucal materno, que poderá se reverter em redução de intercorrências durante o período gestacional devido às infecções bucais. Como avaliações futuras, é possível que o Projeto de Extensão TRA, possa contribuir para discussões científicas acerca do trabalho multidisciplinar neste grupo específico das gestantes.

Com resultados relevantes na redução e controle da doença cárie na população atendida pelo Projeto de Extensão TRA, parece-nos lícito sugerir que as políticas públicas incluam o protocolo TRA, preconizado pela OMS como indispensável nas ações em saúde bucal, principalmente na Atenção Básica. A carência da assistência em saúde nas populações contempladas no Projeto de extensão reflete a necessidade de maior investimento de recursos nessas regiões, valorizando a importância da presença de profissionais de odontologia, devidamente capacitados, para atuar em equipes multiprofissionais das Estratégias de Saúde da Família.

Desta forma, a experiência do Projeto TRA pode ser extrapolada para outros projetos de extensão relacionados à saúde, onde uma ação assistencial evolui para capacitação, pesquisa, literatura e ação multidisciplinar.

\section{Referências Bibliográficas}

CAMARGO, Lucila Basto et al. E-learning used in a training course on atraumatic restorative treatment (ART) for Brazilian dentists. Journal of dental education, v. 75, n. 10, p. 13961401, 2011.

FRENCKEN, Jo E. Evolution of the the ART approach: highlights and achievements. Journal of Applied Oral Science, v. 17, n. SPE, p. 78-83, 2009.

FRENCKEN, Jo E.; HOLMGREN, Christopher J. Atraumatic restorative treatment (ART) for dental caries. STI book, 1999.

FRENCKEN, Jo E.; LEAL, Soraya Coelho; NAVARRO, Maria Fidela. Twenty-five-year atraumatic restorative treatment (ART) approach: a comprehensive overview. Clinical oral investigations, v. 16, n. 5, p. 1337-1346, 2012. 
HOF, Martin A. et al. The Atraumatic Restorative Treatment (ART) approach for managing dental caries: a meta-analysis. International dental journal, v. 56, n. 6, p. 345-351, 2006.

HOLMGREN, C. J.; ROUX, D.; DOMÉJEAN, S. Minimal intervention dentistry: part 5. Atraumatic restorative treatment (ART)-a minimum intervention and minimally invasive approach for the management of dental caries. British dental journal, v. 214, n. 1, p. 11-18, 2013.

MASSONI, A. C. L. T.; PESSOA, Caroline Pereira; OLIVEIRA, Andressa Feitosa Bezerra. Tratamento restaurador atraumático e sua aplicação na saúde pública. Rev Odontol UNESP, v. 35, n. 3, p. 201-7, 2006.

MICKENAUTSCH, Steffen; FRENCKEN, Jo E.; VAN'T HOF, Martin. Factors inhibiting the implementation of the Atraumatic Restorative Treatment approach in public oral health services in Gauteng province, South Africa.Journal of Applied Oral Science, v. 15, n. 1, p. 1-8, 2007.

MICKENAUTSCH, Steffen; GROSSMAN, Elly. Atraumatic Restorative Treatment (ART): factors affecting success. Journal of Applied Oral Science, v. 14, n. SPE, p. 34-36, 2006.

MOLINA, Gustavo Fabián; CABRAL, Ricardo Juan; FRENCKEN, Jo E. The ART approach: clinical aspects reviewed. Journal of Applied Oral Science, v. 17, n. SPE, p. 89-98, 2009. 